\title{
Long Offset Acquisition in Brazil - Looking Deep
}

\author{
Manuel Beitz*, PGS, Jaime Ramos-Martinez, PGS, Joao Victor Lima, PGS, Yermek Balabekov, PGS
}

Copyright 2021, SBGf - Sociedade Brasileira de Geofísica

This paper was prepared for presentation during the $17^{\text {th }}$ International Congress of the Brazilian Geophysical Society held in Rio de Janeiro, Brazil, 16-19 August 2021.

Contents of this paper were reviewed by the Technical Committee of the $17^{\text {th }}$ International Congress of the Brazilian Geophysical Society and do not necessarily International Congress of the Brazilian Geophysical Society and do not necessarily
represent any position of the SBGf, its officers or members. Electronic reproduction or storage of any part of this paper for commercial purposes without the written consent of the Brazilian Geophysical Society is prohibited.

\begin{abstract}
Planning of seismic data acquisition in offshore Brazil needs to address some unique geological and operational challenges, especially when adapting the acquisition for presalt targets. We present findings from a recent survey design study where a complex geological model was built to estimate required offsets for velocity model building through Full Waveform Inversion (FWI). Furthermore, we introduce acquisition concepts that enable the acquisition of densely-sampled near offset data for subsurface imaging, while simultaneously providing sparsely-sampled far-offset data for velocity model building with a minimal environmental footprint.
\end{abstract}

\section{Introduction}

The geology in offshore Brazil poses various challenges to seismic data acquisition. Any seismic survey conducted in this area needs to be carefully planned and adapted to large salt structures, combined with deep sedimentary basins and volcanics. These challenges set the background for very deep reservoirs with variable properties and deeply-buried kitchens.

In contrast to many salt dominated provinces, salt bodies in Brazil have large vertical and lateral extent, with less overhangs or detached small salt structures in the shallow overburden. Unusually, the base of salt is commonly associated with a vertical velocity increase rather than the velocity inversion common to most other global settings. Furthermore, deep sedimentary basins located between large salt structures often show a compaction trend leading to velocity inversions at the top of salt. Pereira et al. (2019) elaborate in detail on the difficulties of presalt reservoir imaging in offshore Brazil.

The aforementioned factors must be sufficiently addressed by new seismic acquisition, either seafloor node or towed streamer, which aim to image presalt geology, rendering pre-survey design a crucial tool for successful data acquisition.

In this study, we present results from a survey design study, aimed to specifically address challenges connected to transmission $\mathrm{FWI}$ and propose streamer acquisition surveys that allow to acquire very long offset data to help accurate velocity model building. We built a model that incorporates some of the geological features discussed above and used it to simulate full wavefield seismic records. We show FWI kernels and depth migrated images to support the proposed acquisition geometries.

\section{Model Building}

Any survey design is only as good as the models it is based on. It is therefore particularly important to ensure the main challenges in the survey area are captured in model building. At the same time, model complexity needs to be adapted to the task at hand to allow discrimination between different factors influencing data quality. A model to investigate FWI update depth does not require a highly detailed reservoir section, but any investigation of resolution requires inclusion of detailed model structures, and 4D modeling requires a realistically behaving reservoir. We decided therefore to build several modified variations of a single structural background framework, each adequately adapted to the challenge. The model presented in Figure 1 was used to generate very long-offset synthetic data used to estimate the required offsets for transmission $\mathrm{FWI}$ model updates.

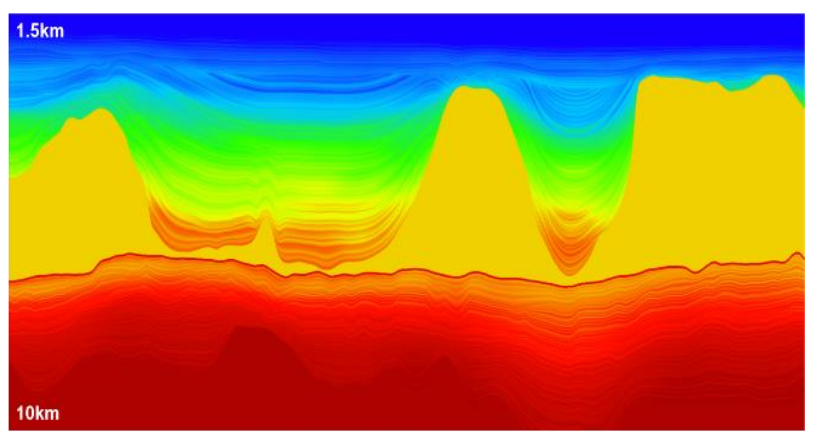

Figure 1: Synthetic model built to investigate the offsets for transmission FWI. Large-scale salt bodies, combined with deep and highly compacted basins, are representative of geology in offshore Brazil. This model is based upon structures found in the Santos Basin.

The model is based upon smooth migration velocities used for imaging of vintage data in Santos Basin. Reflectivity has been introduced using kriging of reflectivity along guiding horizons, resulting in densely spaced reflectors simulating geological structures and unconformities. Different depth sequences have been manipulated separately to reproduce highly compacted sedimentary basins and the resulting velocity inversion of underlying salt. While the model shows discrete layers, volcanics, clastics or detailed reservoir sections have not been included, since the focus was on regional geology and less on specific structures. Too much detail at this stage can be detrimental to the investigation as it has the potential to distract from the effects being investigated. 


\section{Offset Tests}

The simplest way to assess possible penetration depth of FWI model updates with increasing offset is by examining FWI sensitivity kernels. Velocity model building using FWI is commonly based on both, refractions, and reflections but, as this investigation is geared towards very long offsets, we focus on transmission waves. Transmission wave driven $\mathrm{FWI}$ is less sensitive to phenomena that cannot be perfectly reproduced by acoustic modelling engines and therefore considered more robust in very complex geological settings, such as the salt structures found offshore Brazil. Generating a low frequency velocity model under simplified assumptions, which serves as a starting model to stabilize crucial refinements using reflections, is a powerful approach to build stable and detailed velocity models in areas with complex geology.

In order to investigate penetration depth of transmission waves, FWI kernels are generated for a given velocity model and a single source-receiver pair, separated by the offset of interest. This is achieved by first modeling synthetic data for the given source-receiver pair, and subsequently by conducting a single FWI iteration. The resulting velocity model perturbations directly show where the model has been updated.

Figure 2 and Figure 3 show FWI sensitivity kernels generated for the model introduced above. Inspecting the resulting FWI kernels allows us to conclude that a sourcereceiver offset of $10 \mathrm{~km}$ (Figure 2) results in updates reaching well into the shallow salt and deeper sediment basins. However, this penetration is not sufficient to deliver updates from the presalt section.

Increasing offsets to $20 \mathrm{~km}$, as shown in Figure 3, improves penetration depths significantly, with the updates reaching well into the presalt section. Modeling locations have been selected to include significant portions of salt, as well as deep sedimentary basins. The penetration depth is similar for all modeled cases, indicating that the ability to generate presalt velocity updates is independent of the overlying geology.

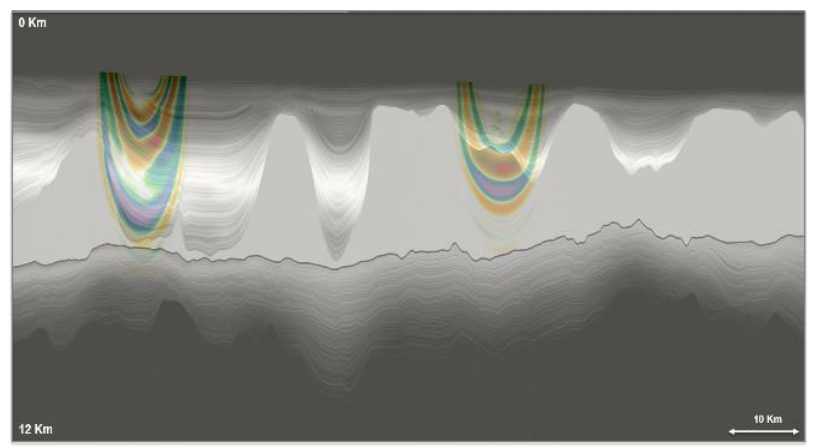

Figure 2: FWI update kernels (color scale) overlaying the velocity model (grey scale). Kernels have been generated for $10 \mathrm{~km}$ offset. While the FWI update kernels reach into salt bodies and deeper basins, updates in the presalt section cannot be achieved.

Source-receiver offsets of up to $10 \mathrm{~km}$ are well within the range commonly achieved by modern towed-streamer surveys using single vessels. Larger offsets require operationally and economically more-challenging geometries employing a larger number of vessels to increase the maximum source-receiver distance beyond the current limits of towed-streamer technology. Seafloor nodes have gained importance in the race to achieve longer offsets, but such acquisition is time-consuming and expensive, particularly in deep water. Apart from increased cost and effort, environmental limitations can also hinder very large-offset configurations, as they often demand a larger source effort.

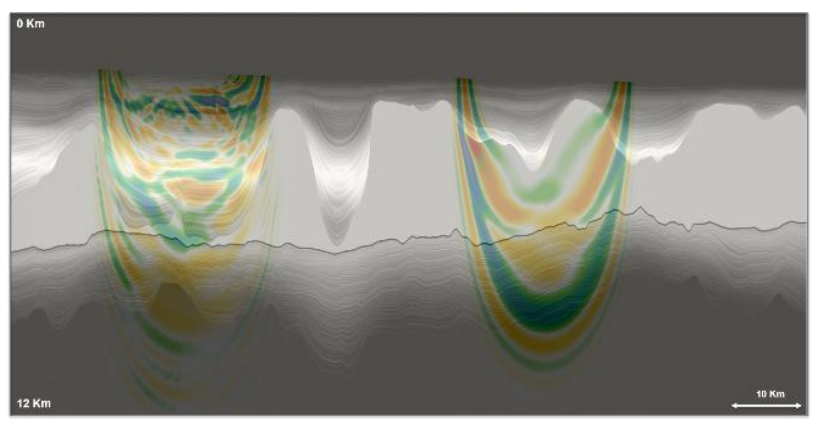

Figure 3: FWI update kernels generated for $20 \mathrm{~km}$ offset. These kernels reach beyond the base of salt, and well into the basement. It can therefore be expected that meaningful velocity updates below salt with transmission FWI are achievable.

Our modeling results show that FWI kernels for offsets of $20 \mathrm{~km}$ are reaching well beyond the base of salt. This suggests that velocity updates of good quality using diving and refracted waves can be obtained using towed streamers if more sophisticated vessel geometries are employed. However, with environmental restrictions in place and the aspiration to reduce operational and financial effort in mind, it is paramount to identify configurations that provide a good image of the subsurface while delivering the offsets sufficient for transmission FWI model building with the least cost.

\section{Offsets for Imaging}

Good understanding of the contribution of different offset classes to the final image can have a major impact on survey economy and image quality. Often, presalt targets are considered subject to limited illumination, assuming that large salt structures are casting strong shadows. Increasing offsets has been used as a remedy to enable undershooting of the shallow structures.

To better understand the contribution of short offsets, we use an image generated with one-way wave equation migration and conventional offsets of $8 \mathrm{~km}$. The image is based upon one synthetic sail-line, assuming that the key challenge can be simulated in 2D. Data were generated using variable density, but no anisotropy, to further isolate acquisition-related effects. The resulting data were migrated with the exact input model to reduce image artifacts associated with imperfect acquisition. In a second exercise, a deliberately modified model was used to reproduce image imperfections observed on real data. 


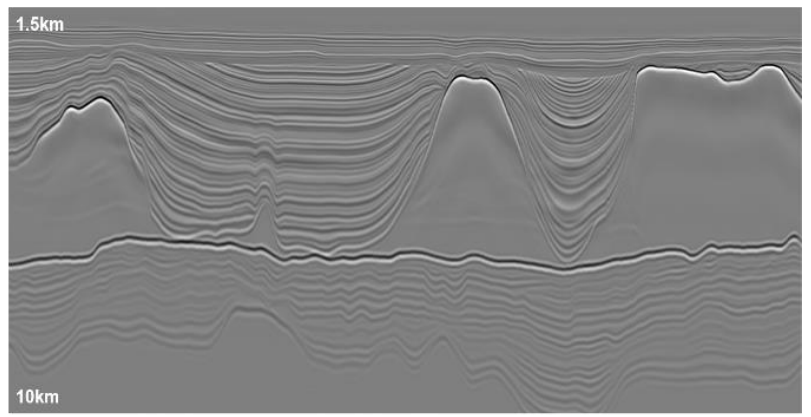

Figure 4: One-way wave equation migration stack image using the exact velocity model. Shot records with offsets of up to $8 \mathrm{~km}$ served as input to migration. Reflections show very little lateral amplitude variation, while some dimming on salt flanks and other offset-related effects can be observed.

The final depth-migrated image using the exact model is presented in Figure 4. Although relatively well-defined, some dimming of salt flanks indicates inadequate maximum offsets. However, continuity of reflections in the presalt section, as well as only minor lateral amplitude variations, indicates a better-than-expected result considering the limited offset range. All geological structures included in the original model are imaged at the correct depth with limited lateral fading of amplitudes or disrupted reflections.

Generating a good image with the exact model is a good start, but it is important to test that the same model with some variations can also be used to reproduce a realistic image. Using an iterative approach, the initial velocity model was degraded and used in migration of the same input data. Figure 5 shows the modified starting model. The top of salt was modified by smoothing some small local features, velocity inversions above salt were removed, as was the high velocity layer below salt. Velocities in the overburden were reduced by $5 \%$, and basement velocities increased by $5 \%$. The resulting model was laterally smoothed with a $2 \mathrm{~km}$ filter length.

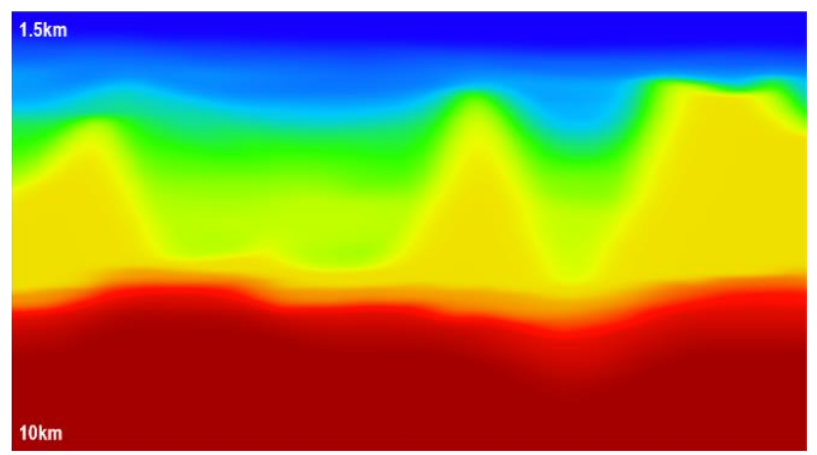

Figure 5: Degraded velocity model generated by modifying the original velocity model. The top of salt was changed, velocities in overburden and basement were modified, and velocity inversions removed. The model was subsequently smoothed.

The original synthetic data with $8 \mathrm{~km}$ offsets were migrated using this degraded model. The resulting image in Figure 4 shows many realistic effects observed on field data after depth migration. The base of salt is discontinuous and shows strong amplitude variations. Areas of strong amplitude diming can be observed below salt flanks and reflection packages in the basement are broken up. Salt flanks are diffuse, and amplitude variations at the top of salt, as well as low frequency artifacts in the salt itself, also indicate incorrect velocities in the overburden and inaccurate salt interpretation.

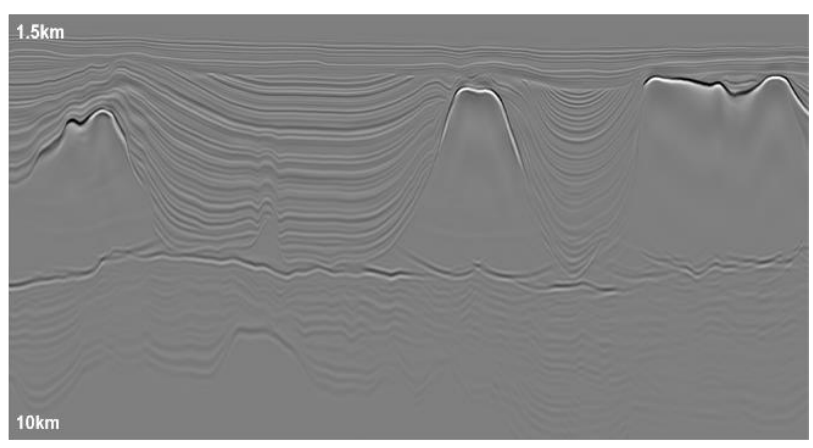

Figure 6: One-way wave equation migration data using a degraded version of the initial velocity model. Appearance of the image is comparable with real data showing broken-up reflection packages and strong lateral amplitude variations.

These results indicate that image quality can be improved to a large extent by application of the correct velocity model. Amplitude dimming, initially accredited to illumination effects, may potentially be the result of defocusing of energy that can be healed by a refined velocity model. This emphasizes the need of performing accurate velocity model building to improve the seismic image.

\section{Long Offset Acquisition}

As noted, Figure 2 shows FWI kernels for offsets of up to $10 \mathrm{~km}$, with insufficient updates in the depths below intrasalt basins. This indicates that conventional towed streamer acquisition using a single vessel will not deliver the offsets necessary to result in transmission FWI updates in the presalt section and velocity model building relies on reflection updates only. However, FWI kernels in Figure 3 reach well into the presalt, suggesting that offsets of up to $20 \mathrm{~km}$ might be sufficient to enable model improvements below salt. Towed-streamer technologies are available that, with some slight modifications, can allow acquisition of the required offsets in a cost-effective manner. Figure 7 presents a selection of acquisition configurations that provide increasingly long offsets with relatively small operational effort. They have been displayed from left to right with increasing complexity. 


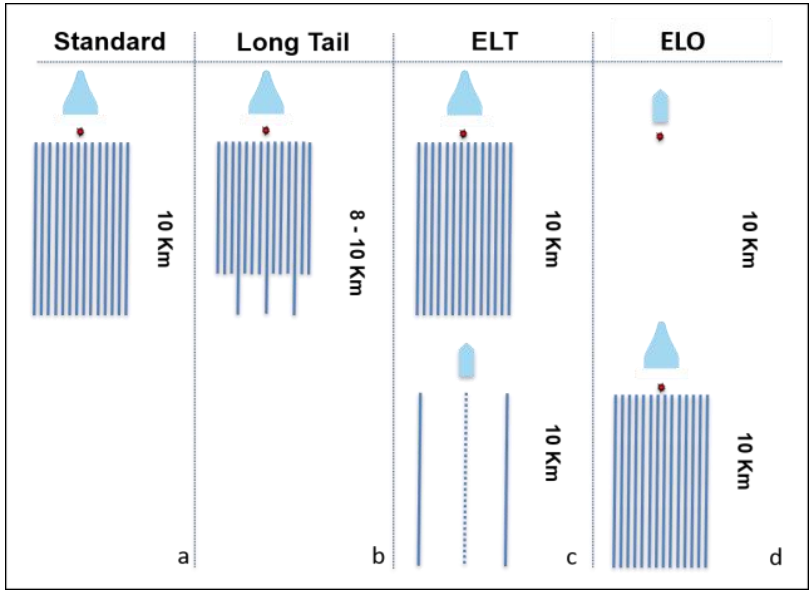

Figure 7: Acquisition geometries currently employed by PGS. a) Standard towed streamer with single vessel; b) Streamer configuration with long tails; c) Extended Long Tails (ELT); d) Extended Long Offset (ELO).

Migration of conventional offsets data as shown in Figure 4 indicates that longer offsets are not required for the construction of a final image if a velocity model of sufficient quality is available. While sufficient energy for imaging can be collected on conventional offsets of up to $8 \mathrm{~km}$, very long offsets are more important for velocity model building than for the final image. As stable FWI updates without cycle-skipping benefit from low frequency data, the sampling of ultra-long offsets can be sparser for (low frequency) model building than the dense near-offset sampling necessary for higher-frequency shallow imaging.

Figure 7 shows some configurations that have been geared towards long offsets and are achievable with available towed streamer technology. The single vessel configurations in Figures $7 \mathrm{a}$ and $7 \mathrm{~b}$ allow acquisition of offsets up to $10 \mathrm{~km}$ using a single vessel. Extended Long Tails (ELT), and Extended Long Offsets (ELO) (options c and d) can provide ultra-long offsets beneficial to transmission FWI updates in the presalt section. These examples are not exhaustive but were identified as fulfilling both requirements of being economically feasible and environmentally friendly. Cost efficiency and relatively small environmental footprint allows these configurations to be deployed on single or multiple azimuths to further improve illumination where warranted by complex geology.

Options b, c and d in Figure 7 use the concept of sparsely sampled far offsets. Long Tail acquisition extends the maximum offsets by including additional long streamer tails behind a conventional streamer spread without deploying additional sources. The long streamer tails record energy emitted by the source towed by the streamer vessel in a more sparsely sampled subset of extended streamers, thereby achieving the required offsets range (Widmaier et al., 2019).

The concept drawing in Figure 8 presents a detailed depiction of an extended Long Tail (ELT) acquisition geometry, wherein sparsely distributed streamers are towed by a second vessel, thus exploiting the long tail concept and the reduced sampling requirements for deep velocity model building. While this configuration shows an offset gap between the forward and rear streamer spreads, continuous offset coverage could be achieved by alternatively deploying the sparse streamer spread ahead of the NAZ spread.

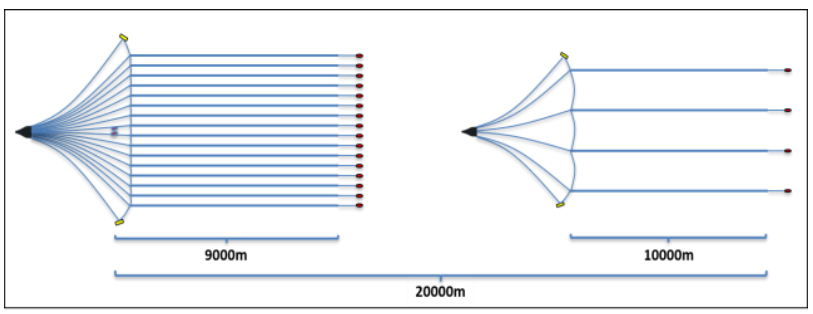

Figure 8: Concept drawing of ELT acquisition geometry with a vessel towing a sparse streamer spread behind a conventional towed streamer configuration.

Reduced environmental impact for the ELT configuration in Figure 7 is realized by adding streamers instead of sources. The sound emissions levels of an ELT survey are the same as a conventional single vessel towed streamer survey ( $a$ and $b$ in Figure 7). Operational and economical effort furthermore can be reduced by deploying the sparse spread from a smaller vessel, since less equipment must be towed through water.

The Extended Long Offset (ELO) concept illustrated in Figure 9 uses a single source towed ahead of a conventional streamer configuration. This setup has been successfully deployed on several occasions, either with unblended or blended (simultaneously actuated) sources. Notably, the reduced sampling requirements of velocity model building methods such as FWI can be exploited to significantly increase the shot point spacing of the forward vessel. This has several advantages over conventional Simultaneous Long Offset (SLO) surveys that use blended shooting schemes. Sparser shot sampling on the far source will contaminate a smaller number of shot records, thereby reducing overall noise levels. Conventional deblending algorithms using coherent energy in different data domains may require some modification. Note that far source data can be used for FWI model building, even when blended (Chemingui et al., 2019).

The reduced number of far offset shots also significantly reduces the environmental footprint in terms of (cumulative) sound exposure level (SEL), and a longer charging time for source arrays might create opportunities for more cost-effective source vessels. It is also possible to employ sources that are tuned to the low frequency range required for $\mathrm{FWI}$ processes. This technology has recently found its first successful application in a commercial survey, offshore Egypt. Long offset data recorded from a lower frequency source concept were acquired with a sparser shot point interval than for the near offsets, and the far offsets were exclusively used for FWI model building in the manner described here. 

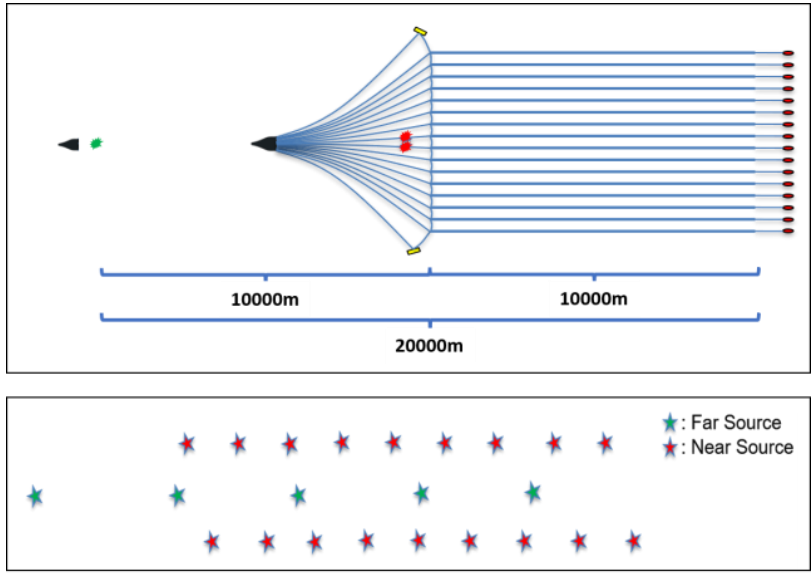

Figure 9: Sparse ELO concept where the far source (green) fires on much sparser shot points than the near sources (red).

\section{Conclusions}

We used a velocity model based on the complex geology in Santos Basin, offshore Brazil, to show with FWI kernels that velocity model updates, using transmission waves, are expected from presalt geology if maximum offsets of up to $20 \mathrm{~km}$ are available. Migrated synthetic data modeled with conventional offsets of up to $8 \mathrm{~km}$ results in a good image with continuous presalt reflections and littleto-no amplitude diming, if the exact velocity model is known. However, some illumination effects can be observed on salt flanks for maximum offsets of $8 \mathrm{~km}$. Migration of the same data with a deliberately degraded velocity model results in reductions in image quality like those found on real data. This indicates that significant image quality improvements on real data should be achievable if the acquisition configuration can enable a high-quality velocity model, based on transmitted waves with subsequent refinements using reflection $\mathrm{FWI}$, and complemented by improved far-offset illumination.

Velocity model building methods such as FWI allow significantly relaxed spatial sampling at long offsets because lower frequencies are required than for imaging. Near offset data used for imaging require dense spatial sampling, but far offset data, only used for velocity model building, can be acquired with much sparser spatial sampling.

Two different methodologies referred to as ELT (Extended Long Tails) and ELO (Extended Long Offset) are identified to allow acquisition of very-long offset data with currently available techniques. These methods reduce survey cost and environmental footprint, courtesy of survey designs that exploit sparser spatial sampling at far offsets. Deep transmission driven FWI model updates, refined by high frequent reflection FWI updates, and complemented by improved illumination of salt flanks will translate to significant image quality improvements in very cost-effective and practical ways without necessitating seafloor acquisition designs that carry much higher costs and larger environmental footprint.

\section{Acknowledgements}

The authors would like to thank PGS for allowing to publish this work.

\section{References}

Chemingui, N., Valenciano, A. [2019] Full Waveform Inversion of Simultaneous Long-Offset Data. 81st Conference and Exhibition, EAGE, Extended Abstracts, Tu R08 13.

Pereira, C. E., Paes, M., Abreu, C. E., Silvany, P., Correa, R. [2019] Benefits of a Multi-Azimuthal data to reservoir characterization: A Brazilian Pre-Salt Case. 16th international Congress, SBGf.

Widmaier, M., O'Dowd, D., Roalkvam, C., [2019] Redefining marine towed-streamer acquisition. First Break, 37(11), 57-62. 